\title{
Pediculose: fatos históricos sobre a doença e a busca persistente pelo tratamento ideal
}

\author{
Pediculosis: a very old disease and the search for an ideal treatment persists \\ Pediculosis: hechos históricos sobre la enfermedad y la búsqueda persistente de \\ tratamiento ideal
}

Fabiana Soares Morgado Garzoni ${ }^{1}$, Vânia Gameiro de Carvalho ${ }^{1 *}$.

\begin{abstract}
RESUMO
Objetivo: Realizar uma revisão sobre o histórico da Pediculose e seu tratamento, desde seu surgimento até os dias atuais. Revisão bibliográfica: A infecção pelo piolho é conhecida pelo homem há milhares de anos, e desde então, vem causando sérios problemas de saúde, como prurido intenso, impetigo, assim como um impacto emocional no paciente infectado. Vários artigos relatam os primeiros achados da Pediculose no mundo, assim como a tentativa de vários tratamentos, desde os mais antigos até o que há de mais atual na literatura, visando erradicar o parasita, e minimizar as consequências na pessoa afetada. Considerações finais: Embora a Pediculose exista há milhares de anos, vários tratamentos já foram propostos, porém, nenhum garante completa eficácia, nem mesmo os mais atuais. Sendo assim, tornam-se imprescindíveis estudos contínuos, visando controlar e erradicar por completo essa ectoparasitose, a fim de evitar graves problemas para a saúde pública e principalmente para as crianças afetadas.
\end{abstract}

Palavras-chave: Pediculose, Piolhos da cabeça, Tratamento.

\begin{abstract}
Objective: To carry out a review of the history of Pediculosis and its treatment, from its emergence to the present day. Bibliographic review: Lice infection has been known to man for thousands of years, and since then, it has been causing serious health problems, such as intense itching, impetigo, as well as an emotional impact on the infected patient. Several articles report the first findings of Pediculosis in the world, as well as the attempt of various treatments, from the oldest to the most current in the literature, aiming to eradicate the parasite, and to minimize the consequences on the affected person. Final considerations: Although Pediculosis has existed for thousands of years, several treatments have been proposed, however, none guarantee complete effectiveness, not even the most current ones. Therefore, continuous studies are essential, aiming to completely control and eradicate this ectoparasitosis, in order to avoid serious problems for public health and especially for the affected children.
\end{abstract}

Keywords: Pediculosis, Head lice, Treatment.

\section{RESUMEN}

Objetivo: Realizar una revisión de la historia de la pediculosis y su tratamiento, desde su aparición hasta la actualidad. Revisión bibliográfica: La infección por piojos es conocida por el hombre desde hace miles de años, y desde entonces ha estado causando serios problemas de salud, como picazón intensa, impétigo, así como un impacto emocional en el paciente infectado. Varios artículos reportan los primeros hallazgos de pediculosis en el mundo, así como el intento de diversos tratamientos, desde los más antiguos hasta los más actuales en la literatura, con el objetivo de erradicar el parásito y minimizar las consecuencias en la persona afectada. Consideraciones finales: Aunque la pediculosis existe desde hace miles de años, ya se han propuesto varios tratamientos, sin embargo, ninguno garantiza una efectividad total, ni siquiera los más actuales. Por ello, los estudios continuos son fundamentales, con el objetivo de controlar y erradicar por completo esta ectoparasitosis, con el fin de evitar problemas graves para la salud pública y especialmente para los niños afectados.

Palabras clave: Pediculosis, Piojos de la cabeza, Tratamiento.

${ }^{1}$ Centro Universitário Municipal de Franca (Uni-FACEF), Franca - SP. *E-mail: vania_gameiro@hotmail.com 


\section{INTRODUÇÃO}

A pediculose do couro cabeludo é uma infestação parasitária causada pelo parasita hematófago Pediculus humanus capitis, popularmente conhecido como piolho, tendo como único hospedeiro o homem (CANDY K, et al., 2020). Trata-se de uma ectoparasitose de grande impacto na saúde pública mundial, já que afeta centenas de milhões de pessoas em todo mundo, incluindo países desenvolvidos, como França, Reino Unido, Suécia, dentre outros, especialmente crianças em idade escolar e seus familiares, devido à facilidade de contágio (BOHL B, et al., 2015; MUMCUOGLU KY, et al., 2021).

O piolho é um inseto de aproximadamente $3 \mathrm{~mm}$, que possui patas para facilitar sua movimentação no couro cabeludo e não possui asas. Seu desenvolvimento ocorre basicamente em 3 fases: fase de ovo (lêndeas), cujo tamanho é menor que $1 \mathrm{~mm}$ e eclode em cerca de 10 dias; fase de ninfa, logo após a eclosão do ovo, perdurando nesta fase em média 10 dias; e finalmente, fase adulta, na qual se diferencia em macho ou fêmea, esta última podendo colocar até 300 ovos durante seu ciclo de vida, com média de 7 a 10 ovos diários (MARINHO LAC, 2015; BARBOSA JV e PINTO ZT, 2003).

Para alimentar-se, o piolho injeta na pele do couro cabeludo sua saliva, causando vasodilatação e reação anticoagulante nesse local, além da reação de sensibilização, provocando o principal sintoma, o prurido, principalmente na região occipital e retroauricular, embora existam relatos de pessoas assintomáticas. Sua transmissão acontece principalmente por contato direto entre cabelos, porém, esse contágio também pode ocorrer através de uso compartilhado de escovas de cabelo, pentes, chapéus, bonés e demais objetos de pessoas infestadas, além de compartilhamento de camas, principalmente em domicílios com número elevado de habitantes, condições socioeconômicas precárias e baixo acesso à assistência de saúde (CARVALHO VO, 2019; MUMCUOGLU KY, et al., 2021).

O Diagnóstico mais seguro é o achado de piolhos vivos na cabeça, entretanto, estes ectoparasitas preferem locais escuros, e pelo fato de serem de cor translúcida e poderem rastejar-se rapidamente, pode tornar difícil a sua visualização. Além disso, pentes finos também podem ser usados para facilitar a visualização de piolhos e lêndeas, que ficam firmemente aderidos à haste capilar, próximo ao couro cabeludo (FRANKOWSKI BL e BOCCHINI Jr JA, 2010; PIMENTA R, et al., 2020).

A pediculose pode gerar, além do intenso prurido, lesões causadas pelo ato de coçar, facilitando o surgimento de infecção secundária, impetigo, pioderma, e, até, edema em gânglios linfáticos retroauriculares. Em infestações maiores podem ocorrer anemia e febre, além de intenso desconforto e sofrimento aos infectados. Além disso, o piolho pode ser um vetor para outras enfermidades graves, como a febre tifóide endêmica. Essa ectoparasitose pode atingir todas as classes sociais, devido à facilidade de transmissão entre pessoas. Como consequências, gera sofrimento, alteração no sono e de concentração, vergonha, angústia e tensões nos infectados, além do impacto educacional, já que muitas escolas proíbem a criança infestada com piolhos de frequentar as aulas até que o problema tenha sido resolvido (BARBOSA JV e PINTO ZT, 2003; FELDMEIER H, 2014; MUMCUOGLU KY, et al., 2021).

Embora mundialmente conhecida há milhares de anos, a pediculose ainda afeta milhões de pessoas, atraindo uma maior atenção da parte médica e científica, já que sua prevalência vem aumentando pelo planeta, principalmente nos últimos 50 anos. Tal fato deve-se principalmente ao desenvolvimento de resistência pelos piolhos aos fármacos amplamente utilizados para controle e tratamento da doença (KOVALICZN RA, et al., 2009).

Dessa maneira, tratando-se de uma enfermidade presente há muito tempo, e que permanece até os dias atuais, o objetivo deste trabalho é descrever o histórico dessa enfermidade, os principais tratamentos utilizados desde os primeiros relatos da doença até os mais empregados atualmente, visando maior conhecimento acerca da dificuldade do controle e da eliminação desta infestação.

\section{REVISÃO BIBLIOGRÁFICA}

Os piolhos de cabeça existem há milhares de anos. Os mais antigos documentados são lêndeas encontradas presas a cabelos de uma pessoa que viveu há aproximadamente 9000 anos na Caverna de 
Nahal Hemar, próximo ao Mar Morto. Além destes achados, também foram relatados a presença de ovos e do próprio Pediculus humanus capitis em múmias egípcias do século IV d.C. e também em índios précolombianos mumificados no Peru datados de 1000-1250 d.C., assim como em outra múmia de 3800 anos na China. Além disso, pentes contaminados datados de aproximadamente 2000 anos foram recuperados em escavações nos desertos da Judéia e Israel (EWING HE, 1924; ZIAS J e MUMCUOGLU KY, 1991; REINHARD KJ e BUIKSTRA J, 2003).

Existem descrições das primeiras tentativas de controle desta parasitose, como fez Heródoto no século $\mathrm{V}$ a.C., através da depilação do cabelo do couro cabeludo, a fim de evitar infestações. Já Celso, no século 30 d.C, propôs o uso tópico de sandarah (substância obtida de uma árvore africana) e de uma planta trepadeira da família do inhame adicionada a óleo e vinagre, visando o controle da infestação. O caldo de víbora foi sugerido por Pliny em 23-79 d.C para ser aplicado nas partes afetadas pelo ectoparasita, enquanto Galeno (121-201 d.C) utilizou ervas medicinais como sementes de stavesacre e Delphinium sp contendo alcaloides. Uma combinação de mercúrio e óleo de rosas foi usada por Avicena (980-1037 d.C) (MUMCUOGLU KY, 1996).

No século XVI, Amato Lusitano, médico português, relatou que o único tratamento utilizado era a remoção mecânica dos ectoparasitas, vindo daí a expressão conhecida como: "catar piolho" (FIGUEIREDO TIC, 2013). A planta Chrysanthemum cinerariaefolium (Treviranus), conhecida por suas propriedades inseticidas desde os tempos da Pérsia, começou a ser utilizada na Europa no século XIX, com posterior extração de suas substâncias ativas (Piretrinas). Em 1948 foi descoberto o inseticida DDT (Dicloro Difenil Tricloroetano) para controle de piolhos e este foi amplamente utilizado com esse fim pela primeira vez em 1939, durante a $2^{\mathrm{a}}$ Guerra Mundial (MUMCUOGLU KY, 1996). Embora ainda utilizado em alguns países em desenvolvimento, seu uso atualmente não é mais recomendado, principalmente devido a problemas ambientais relacionados ao DDT (FRANKOWSKI BL e BOCCHINI JR JA, 2010).

Atualmente, existem várias maneiras de controlar e tratar a Pediculose. Infelizmente, nenhuma apresenta $100 \%$ de eficácia, principalmente nos últimos anos, já que a presença de piolhos resistentes a alguns medicamentos é relatada desde meados da década de 1990, devido ao uso inadequado e excessivo dos pediculicidas de ação neurotóxica (FELDMEIER H, 2014; FIGUEIREDO TIC, 2013). O diagnóstico correto, a forma de administração, dosagem, excipientes da fórmula, adesão ao tratamento, reinfecção, ocorrência de morte dos ovos e propriedades residuais do fármaco aplicado interferem diretamente na eficácia ou não do tratamento (FRANKOWSKI BL e BOCCHINI JR JA, 2010; BURGESS IF, 2009; HUSNI L e AL-WAIZ M, 2021).

O tratamento deve ser baseado na eficácia e segurança do produto, dos padrões locais de resistência, facilidade de uso e custo (FRANKOWSKI BL e BOCCHINI JR JA, 2010). Por convenção, o tratamento pode ser dividido em medicamentos tópicos e orais, além da remoção manual e cuidados gerais.

\section{Tratamentos Tópicos}

A Permetrina em loção a $1 \%$ é um piretróide sintético que atua interferindo nas proteínas transportadoras de sódio nos insetos, bloqueando a repolarização desses canais dos neurônios, causando neurotoxicidade, paralisia respiratória e sua posterior morte. Este medicamento começou a ser prescrito no tratamento da Pediculose no ano de 1986 nos Estados Unidos, sendo considerada a substância menos tóxica para os seres humanos, com mínima exposição sistêmica, podendo ocasionar efeitos adversos extremamente raros, como prurido, eritema e edema (FIGUEIREDO TIC, 2013; BURKHART C, et al., 2012).

Já a Permetrina 5\% ainda não foi aprovada pelo Food Drug Administration (FDA) como pediculicida, apenas para tratamento de escabiose, porém, estudos sugerem que pode ser eficiente no tratamento da pediculose contra piolhos resistentes a Permetrina $1 \%$. No entanto, por ser o tratamento mais utilizado mundialmente, e nem sempre utilizado da maneira correta, estudos têm demonstrado a ocorrência da diminuição da eficácia dos Piretroides devido à resistência dos piolhos a esses fármacos, ocasionada principalmente por mutações nos canais de sódio dos insetos, o que reduz, assim, à sensibilidade ao fármaco e diminui a sua eficácia. A Permetrina 1\% pode ser indicada para crianças maiores de 2 meses, gestantes e lactantes (FRANKOWSKI BL e BOCCHINI JR JA, 2010; CÉSAR A, et al., 2017; BURGESS IF, 2009). 
A Deltametrina é um medicamento sintético derivado do ácido crisântemo, que é extraído da flor do crisântemo (Chrysanthemum cinerariaefolium). Seu mecanismo de ação é obtido após a absorção da substância pelo exoesqueleto do piolho, atingindo os gânglios nervosos periféricos e o Sistema Nervoso Central, provocando paralisia e posterior morte do ectoparasita. Este medicamento possui ação seletiva, elevada segurança e é pouco tóxico; seu elevado poder residual provoca alta letalidade do ectoparasita (FERNANDES TF, 2011).

Os Piretróides (Permetrina, Deltametrina, etc), também com ação neurotóxica, foram introduzidos no mercado para tratamento da Pediculose na década de 1980, são inseticidas sintéticos, possuem estruturas semelhantes à Piretrina (componente ativo extraído das flores do Chrysanthemun cinerariaefolium) e vêm sendo aplicados para o controle de insetos há mais de 200 anos. Possuem toxicidade mínima aos mamíferos, alto poder residual e são altamente eficientes (FRANKOWSKI BL e BOCCHINI JR JA, 2010; VIEIRA HP, et al., 2007).

Ademais, os piretróides possuem segurança e absorção mínima pelo paciente, com a descrição de poucos efeitos colaterais como manchas, descamações, edema e prurido. Na maioria das vezes, a Piretrina é vendida associada ao Butóxido de Piperonil (PPB) a 4\%, pois essa formulação reduz a degradação da piretrina, diminuindo a chance de resistência ao medicamento, porém, torna-se ineficaz caso já exista a resistência à Permetrina, sendo indicado para crianças a partir de 2 anos de idade (FIGUEIREDO TIC, 2013; JONES KN e ENGLISH III JC, 2003; FRANKOWSKI BL e BOCCHINI JR JA, 2010; BOHL B, et al., 2015).

Lindano 1\% (Hexaclorocicloexano) é um organoclorado facilmente absorvido pelo exoesqueleto do piolho, ocasionando hiperestimulação dos neurônios, provocando paralisia e morte do ectoparasita. Começou a ser utilizado para o tratamento da pediculose em 1951, porém, devido a sua toxicidade ao sistema nervoso, que pode desencadear convulsões graves nos pacientes e o desenvolvimento de resistência, é recomendado ser utilizado somente como tratamento de segunda linha, ou seja, quando outros tratamentos mais seguros e eficazes já foram realizados sem sucesso (FIGUEIREDO TIC, 2013; BURKHART C, et al., 2012; FRANKOWSKI BL e BOCCHINI JR JA 2010).

Outros efeitos colaterais do Lindano $1 \%$ podem ocorrer, como prurido, edema leve, ardor, formigamentos, eritema e erupção cutânea também foram relatados. Por isso, encontra-se em desuso e é contraindicado em pessoas com histórico de convulsões e principalmente em pessoas com HIV, pois apresentam limiar convulsivo reduzido. Deve ser evitado em neonatos, crianças, idosos, adultos com menos de $50 \mathrm{Kg}$, gestantes e lactantes, pois o medicamento é eliminado no leite materno e foi associado a convulsões e alterações hepáticas no lactente (BOHL B, et al., 2015; JONES KN e ENGLISH III JC, 2003; FRANKOWSKI BL e BOCCHINI JR JA 2010; CESAR A, et al., 2017; CARVALHO VO, 2019).

O Malatião $(0,5 \%)$ é um organofosforado, cuja ação é de ligação (inibição) à acetilcolinesterase do piolho, ocasionando sua paralisia e posterior morte (BURKHART C, et al., 2012). Embora seja considerado altamente eficaz para a eliminação de piolhos e lêndeas, essa substância foi retirada 2 vezes do mercado nos Estados Unidos por ser altamente inflamável e pelo forte odor. Mesmo assim, foi reintroduzido em 1999 como tratamento de segunda linha, sendo indicado apenas em situações de parasitas resistentes aos fármacos de primeira linha. Na Europa, as substâncias terpineol, dipenteno e óleo de agulha de pinheiro foram acrescidas à fórmula do Malatião, com o intuito de aumentar o poder pediculicida e postergar o desenvolvimento de resistência ao produto (FRANKOWSKI BL e BOCCHINI JR JA, 2010; FIGUEIREDO TIC, 2013).

Devido ao fato do Malatião $0,5 \%$ ser altamente inflamável, deve ser cuidadosamente orientado ao responsável quanto aos cuidados durante a aplicação do produto, devendo ser indicado somente para crianças maiores de 6 anos de idade e não é recomendado para mulheres que estão amamentando (FRANKOWSKI BL e BOCCHINI JR JA, 2010; BURKHART C, et al., 2012; CESAR A, et al., 2017).

O Álcool Benzílico 5\% é um produto que foi aprovado para tratamento da pediculose nos EUA em 2009, com ação de impedir o fechamento dos espiráculos respiratórios dos piolhos, obliterando as vias aéreas, ocasionando asfixia e morte desses insetos, sendo, portanto, o primeiro pediculicida com ação não neurotóxica (BOHL B, et al., 2015). Demonstrou mais de 75\% de eficácia em dois estudos clínicos, porém, 
sem efeito ovicida (FERNANDES TF, 2011; HUSNI L e AL-WAIZ M, 2021). Não é recomendado para crianças menores de 6 meses, a fim de evitar grande absorção do produto, além de alguns efeitos indesejáveis, como prurido, eritema e irritações nos olhos. (FRANKOWSKI BL e BOCCHINI JR JA, 2010; BOHL B, et al., 2015; HUSNI L e AL-WAIZ M, 2021).

Spinosad 0,9\%, um novo produto, originado da fermentação do Saccharopolyspora spinosa, primeiramente identificado em 1982, utilizado como inseticida desde 1997, foi aprovado pela FDA somente em 2011 para tratamento da Pediculose (BOHL B, et al., 2015). Possui ação pediculicida, interferindo nos receptores nicotínicos da acetilcolina do piolho, causando a morte dos ectoparasitas e dos ovos. É indicado para crianças maiores de 6 meses, embora alguns autores recomendem apenas para as maiores de 4 anos (FIGUEIREDO TIC, 2013; BOHL B, et al., 2015; YETMAN RJ, 2015).

O Benzoato de Benzila foi utilizado desde o fim da década de 1940 para tratamento da Pediculose e escabiose, sendo considerado, na época, como eficaz, não irritante, econômico e prático ao uso. Porém, atualmente encontra-se em desuso devido à resistência do ectoparasita à substância, além de ocasionar efeitos indesejados no paciente (CARVALHO VO, 2019; FERNANDES TF, 2011).

A Ivermectina loção 0,5\%, aprovada em 2012 pelo FDA, é recomendada para crianças maiores de 6 meses. Possui eficácia contra o ectoparasita quando aplicada em cabelos secos, principalmente nos casos de piolhos resistentes a Permetrina, porém sem efeito ovicida (YETMAN RJ, 2015; FELDMEIER H, 2014). Já Husni L e Al-Waiz M (2021) demonstraram a eficácia da Ivermectina solução a $2 \%$ em seus estudos, apresentando propriedades tanto pedicuicida quanto ovicida, principalmente se realizada 2 aplicações, além de possuir as vantagens de ser barata e quase nenhum efeito colateral.

O Carbamato de Carbaril já foi utilizado na década de 1980 com boa eficácia no tratamento da Pediculose (MAUNDER JW, 1981). Este medicamento age inibindo a acetilcolinesterase do piolho com consequente paralisia e morte, porém existem relatos da existência de efeitos indesejados como náusea, tontura, parada respiratória e até óbito, além de possuir potencial cancerígeno, o que torna a sua utilização não recomendada (LIMA LF, 2017).

Novos estudos estão sendo feitos com substâncias, tais como o Miristato de Isopropila e 1,2-octanodiol, que dissolvem e rompem a cera que cobre a cutícula do exoesqueleto do piolho, o que causa a perda de água pela cutícula e a morte do parasita. Tais substâncias, até o momento, parecem ser seguras, sem efeitos colaterais (FELDMEIER H, 2014).

\section{Tratamento sistêmico}

A Ivermectina é um medicamento anti-helmíntico utilizado no tratamento contra nematelmintos parasitários e artrópodes (insetos, carrapatos e ácaros). Seu mecanismo de ação consiste na imobilização dos parasitas através da ativação de canais de cloro $(\mathrm{Cl})$ controlados por glutamato, presentes somente nos invertebrados, causando hiperpolarização devido ao aumento da concentração do cloreto intracelular das células musculares e nervosas, o que resulta na paralisia do parasita (BURKHART C, et al., 2012; FRANKOWSKI BL e WEINER LB, 2002).

Estudos revelam que não há evidência relevante quanto a maior efetividade da Ivermectina oral versus a tópica ou outro pediculicida, já que ambas são eficazes, sendo indicada a Ivermectina oral associada à tópica somente em casos de infestações mais graves. Porém, apenas a forma tópica desse medicamento foi aprovada pelo FDA para o tratamento da Pediculose. Embora tenha se mostrado segura, com ausência de efeitos colaterais graves, é contraindicada em crianças com menos de $15 \mathrm{Kg}$ (FRANKOWSKI BL e BOCCHINI JR JA, 2010; FRANKOWSKI BL e WEINER LB, 2002; JONES KN e ENGLISH III JC, 2003; YOUNG C e ARGÁEZ C, 2019).

O Sulfametoxazol-Trimetoprima é um antibiótico usado somente nos casos mais graves de infestações ou superinfecção bacteriana associada. Acredita-se que ao sugar o sangue do paciente, o piolho ingere o medicamento, causando eliminação das bactérias simbióticas em seu intestino ou algum outro efeito tóxico. Para uma maior eficácia, é recomendado que seja associado ao tratamento tópico com Permetrina 1\%. Possui 
efeitos colaterais raros, porém, quando presentes, são graves, como por exemplo, necrólise epidérmica tóxica, Síndrome de Stevens-Johnson, anemia aplástica e discrasias sanguíneas. Por essa razão, ainda não foi aprovado pelo FDA como pediculicida (JONES KN e ENGLISH III JC, 2003; FRANKOWSKI BL e WEINER LB, 2002; FRANKOWSKI BL e BOCCHINI JR JA, 2010).

\section{Produtos naturais / ervas medicinais}

Ultimamente, estudos com óleos essenciais têm demonstrado sucesso no tratamento. Estes, são extraídos das plantas como Citrus bergamia, Citronella sp, Eucalyptus sp, Santalum sp, Chrysopogon Zizanioides, Zingiber officinale, Pimpinella anisum, Myristica fragrans, dentre outras. Os óleos essenciais são produzidos geralmente em combinação com várias plantas diferentes até chegar na formulação adequada. Possuem as vantagens de serem pouco tóxicos e não poluentes ao meio ambiente. Porém, deve-se ter cautela, já que há maior risco de desencadear reações alérgicas (CANDY K, et al., 2020).

Existem relatos de medidas caseiras à base de plantas, tais como xampus feitos à base de ervas como Plectrantus barbatus (mais conhecida como Boldo) e Momordica charantia L (conhecida como Melão de São Caetano) e também a erva Ruta graveolens (conhecida como Arruda), além de spray de erva doce e óleo de lavanda. Todas essas medidas, porém, não possuem efeito comprovado no controle da infestação de piolhos, embora já exista relato de um estudo demonstrando certa efetividade da ação da Arruda no controle da pediculose (BARBOSA JV e PINTO ZT, 2003; KOVALICZN RA, et al., 2009; HUSNI L e AL-WAIZ M, 2021).

\section{Tratamento mecânico.}

Isoladamente, a remoção manual de lêndeas e piolhos não é suficiente, porém, após a aplicação tópica do pediculicida sempre é recomendada, já que nenhum medicamento tópico garante a total eliminação dos ectoparasitas e de seus ovos. Além disso, a efetividade da remoção, principalmente utilizando o pente fino depende do formato do pente, assim como da habilidade no uso do acessório utilizado pela pessoa. Há relatos de que produtos, como o vinagre, e géis, que possam facilitar a remoção de lêndeas dos cabelos, porém sem eficácia científica comprovada. Dessa maneira, deve ser considerado um mecanismo complementar para a eliminação dos parasitas e de seus ovos (FIGUEIREDO TIC, 2013; FRANKOWSKI BL e WEINER LB, 2002; MUMCUOGLU KY, et al., 2021).

Foi realizado um estudo com lêndeas tratadas com vinagre, álcool isopropílico, azeite e maionese, que, embora tenha revelado um atraso na taxa de eclosão das lêndeas, não demonstrou redução da mesma (TAKANO-LEE M, et al., 2004). Eletrocussão, um tipo de pente eletrônico, provoca morte dos piolhos ao ser passado nos cabelos secos, pois emite pequenas descargas elétricas quando os dentes metálicos do pente encontram algum piolho. Embora seja utilizado, não há estudos científicos que comprovem e garantam sua eficácia e segurança (FIGUEIREDO TIC, 2013; LIMA LF, 2017). Uma outra maneira de avaliar se as lêndeas estão viáveis ou vazias após o tratamento, é através da Dermatoscopia, método para o acompanhamento da eficácia do tratamento, possuindo as vantagens de ser rápido, indolor e, por isso, possui boa aceitação, principalmente da população pediátrica (PIMENTA R, et al., 2020).

\section{Medidas Sanitárias}

Além da importância do tratamento propriamente dito, faz-se necessário que profissionais da saúde saibam orientar a população em geral, sobre a vigilância constante, medidas de higiene e a respeito dos métodos de prevenção de novos contágios, além de orientar o tratamento eficaz e atualizado, pois o tratamento inadequado é a principal causa de insucesso do tratamento e não a resistência dos piolhos aos pediculicidas (BARBOSA JV e PINTO ZT, 2003; CARVALHO VO, 2019; FRANKOWSKI BL e BOCCHINI JR JA, 2010; CÉSAR A, et al., 2017; FERNANDES TF, 2011; MUMCUOGLU KY, et al., 2021).

Importante salientar que, toda a família deve ser avaliada e, se preciso, tratada adequadamente, até mesmo de forma profilática. As roupas da criança infectada, toalhas, roupas de cama, chapéus, pentes e similares devem ser lavados com água quente. Carpetes e tapetes devem ser aspirados. Itens que não possam ser lavados, devem ser colocados em sacos plásticos fechados por 2 semanas, para que, assim, sejam eliminados tanto os parasitas quanto seus ovos (FRANKOWSKI BL e BOCCHINI JR JA, 2010; FERNANDES TF, 2011; YETMAN RJ, 2015). 


\section{CONSIDERAÇÕES FINAIS}

Diante do que foi abordado, percebe-se que existem dificuldades e limitações para o tratamento da Pediculose, visto que os piolhos já adquiriram resistência à maioria dos fármacos utilizados atualmente. Desta maneira, fica claro a importância da elaboração de novos fármacos eficazes e seguros sobre tratamento e controle da doença, visando alternativas à resistência dos piolhos aos medicamentos utilizados, minimizando possíveis efeitos colaterais. A procura por medicamentos naturais, com mecanismos de ação diferentes dos encontrados hoje em dia pode ser um ponto de partida para futuros estudos sobre o tema. Por fim, ressaltase a importância da orientação aos pais, professores e profissionais de saúde em manter a higiene adequada, além da vigilância a fim de evitar possíveis novas infestações.

\section{REFERÊNCIAS}

1. BARBOSA JV, PINTO ZT. Pediculose no Brasil. Entomol. Vect. 2003; 10 (4): 579-86.

2. BOHL B, et al. Clinical Practice Update: Pediculosis Capitis. Pediatric Nursing. 2015; 41 (5): 227-234.

3. BURGESS IF. Current treatments for pediculosis capitis. Current Opinion in Infectious Diseases. 2009; $22: 131$-36.

4. BURKHART C, et al. As Bases Farmacológicas da Terapêutica de Goodman e Gilman. 12ª ed. São Paulo: Mc Graw Hill, 2012; 1818-19p.

5. CANDY K, et al. Essential Oils as a Potential Treatment Option for Pediculosis. Planta Med, 2020; 86: 619-630.

6. CARVALHO VO. Infecções cutâneas parasitárias: aspectos clínicos e atualização terapêutica. Sociedade Brasileira de Pediatria Departamento de Dermatologia. 2019; 4: 1-10.

7. CÉSAR A, et al. Gravidez, Aleitamento e Fármacos em Dermatologia: Tratamento Tópico. Sociedade Portuguesa de Dermatologia e Venereologia. 2017; 75(1): 19-26.

8. EWING HE. Lice from human mummies. Science. 1924; 60 (1556): 389-90.

9. FELDMEIER H. Treatment of Pediculosis Capitis: A Critical Appraisal of the Current Literature. Am. J Clin Dermatol. 2014; 15: 401-412.

10. FERNANDES TF. In: Pediculose: novas abordagens para uma antiga doença. Sociedade de Pediatria de São Paulo: Disponível em: https://www.spsp.org.br/2011/09/29/pediculose_novas_abordagens_para_uma_antiga_doenca/ Acesso em: 22 de maio de 2020.

11. FIGUEIREDO TIC. Pediculose. Dissertação (Mestrado em Ciências Farmacêuticas) - Universidade Fernando Pessoa, Cidade do Porto, 2013; 4,19,20p.

12. FRANKOWSKI BL, BOCCHINI JR JA. Head Lice. American Academy of Pediatrics, 2010; 126 (2): $392-403$.

13. FRANKOWSKI BL, WEINER LB. Head Lice. American Academy Of Pediatrics, $2002 ; 110$ (3): 638-43

14. HUSNI L, AL-WAIZ M. Topical ivermectin in the treatment of pediculosis capitis. Our Dermatol Online. 2021; 12(1): 1418

15. JONES KN, ENGLISH III JC. Review of Common Therapeutic Options in the United States for the Treatment of Pediculosis Capitis. Clinical Infectious Diseases. 2003; 36 (1): 1355-61.

16. KOVALICZN RA, et al. Avaliação inseticida contra Pediculus humanus capitis De Geer e de irritação cutânea aguda de soluções hidroglicólicas contendo Ruta graveolens L. Rev. Bras. Farm. 2009; 90 (3): 200-203

17. LIMA LF. Gel de Polímero para tratamento da Pediculose. Dissertação (Mestrado Profissional em Ciência, Tecnologia e Inovação) - Universidade Federal do Rio Grande do Norte, Natal, 2017; 27,30p.

18. MARINHO LAC, MILAN EP. Ectoparasitoses. In: Focaccia R, editor. Tratado de Infectologia. 5 ${ }^{\text {a }}$ Ed. São Paulo: Atheneu; 2015; 2145-46p.

19. MAUNDER JW. Clinical and laboratory trials employing carbaryl against the human head-louse, Pediculus humanus capitis (de Geer). Clinical and Experimental Dermatology, 1981;6 (6): 605-12.

20. MUMCUOGLU KY. Control of Human Lice (Anoplura: Pediculidae) Infestations: Past and Present. American Entomologist, 1996; 42: 175-78.

21. MUMCUOGLU KY, et al. International recommendations for an effective control of head louse infestations. International Journal of Dermatology, 2021; 60: 272-280.

22. PIMENTA R, et al. Dermatoscopia na Idade Pediátrica - Parte II: Dermatoses Infecciosas e inflamatórias. Revista da Sociedade Portuguesa de Dermatologia e Venereologia, 2020; 78 (3): 197-207

23. REINHARD KJ, BUIKSTRA J. Louse Infestation of the Chiribaya Culture, Southern Peru: Variation in Prevalence by Age and Sex. Memórias do Instituto Oswaldo Cruz, 2003; 98 (1): 173-79.

24. TAKANO-LEE M, et al. Home Remedies to Control Head Lice: Assessment of Home Remedies to Control the Human Head Louse, Pediculus humanus capitis (Anoplura: Pediculidae). Journal of Pediatric Nursing, 2004; 19 (6): $393-98$.

25. TORQUATO NR, et al. Novas perspectivas do tratamento da Pediculose. e-Scientia, 2019;12 (1): 15-18.

26. VIEIRA HP, et al. Otimização e validação da técnica de extração líquido-líquido com partição em baixa temperatura (ell-pbt) para piretróides em água e análise por cg. Quim. Nova, 2007; 30 (3): 535-40.

27. YETMAN RJ. The Child With Pediculosis Capitis. Journal of Pediatric Health Care, 2015; 29 (1): 118-120.

28. YOUNG C, ARGÁEZ C. Ivermectin for parasitic skin infections of lice: a review of comparative clinical effectiveness, cost-effectiveness, and guidelines. CADTH rapid response report: summary with critical appraisal from Canadian Agency for Drugs and Technologies in Health.2019

29. ZIAS J, MUMCUOGLU KY. Pre-Pottery Neolithic B Head Lice from Nahal Hemar Cave. Atiqot, 1991; 20: 167-68. 